\title{
Stereotactic ablative body radiotherapy for spinal metastasis from hepatocellular carcinoma: its oncologic outcomes and risk of vertebral compression fracture
}

\author{
Gyu Sang Yoo ${ }^{1, *}$, Hee Chul Park ${ }^{1,5, *}$, Jeong Il Yu ${ }^{1}$, Do Hoon Lim ${ }^{1}$, Won Kyung Cho ${ }^{1}$, \\ Eonju Lee ${ }^{6}$, Sang Hoon Jung ${ }^{1}$, Youngyih Han ${ }^{1}$, Eun-Sang Kim², Sun-Ho Lee ${ }^{2}$, Whan \\ Eoh $^{2}$, Se-Jun Park ${ }^{3}$, Sung-Soo Chung ${ }^{3}$, Chong-Suh Lee ${ }^{3}$ and Joon Hyuk Lee ${ }^{4}$ \\ ${ }^{1}$ Department of Radiation Oncology, Samsung Medical Center, Sungkyunkwan University School of Medicine, Seoul, Korea \\ ${ }^{2}$ Department of Neurosurgery, Samsung Medical Center, Sungkyunkwan University School of Medicine, Seoul, Korea \\ ${ }^{3}$ Department of Orthopedic Surgery, Samsung Medical Center, Sungkyunkwan University School of Medicine, Seoul, Korea \\ ${ }^{4}$ Department of Medicine (Division of Hepatology), Samsung Medical Center, Sungkyunkwan University School of Medicine, \\ Seoul, Korea \\ ${ }^{5}$ Department of Medical Device Management and Research, Samsung Advanced Institute for Health Sciences and Technology, \\ Sungkyunkwan University, Seoul, Korea \\ ${ }^{6}$ Department of Radiation Oncology, Samsung Changwon Hospital, Sungkyunkwan University School of Medicine, \\ Changwon-si, Korea \\ *These authors have contributed equally to this work as the first authors \\ Correspondence to: Jeong II Yu, email: jeongil.yu@samsung.com \\ Se-Jun Park, email: sejunos.park@samsung.com \\ Keywords: stereotactic ablative body radiotherapy, spine, hepatocellular carcinoma, vertebral compression fracture \\ Received: March 15, $2017 \quad$ Accepted: July 30, $2017 \quad$ Published: August 24, 2017 \\ Copyright: Yoo et al. This is an open-access article distributed under the terms of the Creative Commons Attribution License 3.0 \\ (CC BY 3.0), which permits unrestricted use, distribution, and reproduction in any medium, provided the original author and source \\ are credited.
}

\section{ABSTRACT}

Spinal metastases from hepatocellular carcinoma (HCC) require high-dose irradiation for durable pain and tumor control. Stereotactic ablative body radiotherapy (SABR) enables the delivery of high-dose radiation. However, but vertebral compression fracture (VCF) can be problematic. The aim of his study is to evaluate the outcome and risk of VCF after SABR for spinal metastasis from HCC. We retrospectively reviewed 33 lesions in 42 spinal segments from 29 patients who received SABR with 1 fraction (16-20 Gy), or 3 fractions (18-45 Gy) from September 2009 to January 2015. The 1-year local control (LC) rate was $68.3 \%$. Radiographic grade of cord compression (RGCC) was the only independent prognostic factor associated with LC ( $P$ $=0.007$ ). The 1-year ultimate LC rate including the outcome of salvage re-irradiation was $87.2 \%$. The pain response rate was $73.3 \%$ according to the categories of the International Bone Metastases Consensus Group. The 1-year VCF-free rate was $71.5 \%$. Pre-existing VCF $(P<0.001)$ and only-lytic change $(P=0.017)$ were associated with a higher post-SABR VCF rate. One-third of post-SABR VCFs required interventions. SABR for spinal metastases from HCC provided efficacious LC, especially for lesions with RGCC $\leq$ II, and showed effective and durable pain relief. As VCF after SABR occurred frequently for vertebral segments with pre-existing VCF and only-lytic change, early preventive vertebroplasty is considerable for those high-risk vertebral segments. 


\section{INTRODUCTION}

Hepatocellular carcinoma (HCC) is the fifth most common cancer worldwide and the second leading cause of cancer-related death [1]. Although distant metastasis is less likely in HCC than other tumors, the incidences have increased in the last decade as the overall survival (OS) of patients with metastatic HCC has improved $[2,3]$. Therefore, efficient palliation of metastasis from $\mathrm{HCC}$ has become an important clinical issue.

Spinal metastasis is estimated to represent $40 \%$ of bone metastases from HCC [4]. Radiotherapy (RT) is the most commonly used modality for spinal metastasis from HCC. RT of 30 Gy in 10 fractions is widely accepted as conventional regimen for spinal metastasis. However, as dose-response relationships between RT dose and symptom palliation have been reported, and conventional RT for spinal metastasis from HCC showed a high retreatment rate of up to $50 \%$, there have been efforts to deliver a higher dose to spinal metastases from $\mathrm{HCC}$ [4-6].

Stereotactic ablative body radiotherapy (SABR) enables delivery of high-dose radiation to a specific target. Due to recent technological advances, SABR is now considered an effective modality for spinal metastasis. However, SABR can result in some orthopedic complications, such as vertebral compression fracture (VCF) or neuropathies [7]. Although there are a limited number of studies showing the effectiveness of SABR for spinal metastasis from HCC in local control (LC) and pain palliation $[8,9]$, there have not been any reports evaluating the risk of VCF in company with the oncologic outcome.

In this study, we aimed to evaluate the oncologic outcome and risk of VCF after SABR for spinal metastasis from HCC. We also identified the prognostic factors related to oncologic outcome and post-SABR VCF.

\section{RESULTS}

\section{Patient and tumor characteristics}

The patient and tumor characteristics are summarized in Table 1. Twenty-five (86.2\%) patients were Child-Pugh classification A. The Cancer of the Liver Italian Program (CLIP) score was 2 or less for 22 patents (75.9\%). Ten patients $(34.5 \%)$ had visceral metastasis additionally. One patient received fixation before SABR. More than half (54.5\%) of the lesions showed radiographic grade of cord compression (RGCC) $\leq$ II. About $64 \%$ of the lesions showed no neurologic symptom (neurological grade a) before SABR. The median age was 55 years (range, 35-74 years). The median tumor volume was 21.3 cc (range, 2.6-221.27 cc). The median follow-up was 7 months (range, 1-43 months).

\section{Oncologic outcomes}

Twenty-five of 33 lesions were evaluable by followup images. Partial remission and stable disease status were achieved for 2 and 16 lesions, respectively. The 6-month and 1 -year $\mathrm{LC}$ rates were $74.5 \%$ and $68.3 \%$, respectively (Figure 1A). Seven of 25 lesions showed local failure during the follow-up. Six of the 7 progressed lesions showed epidural progression near the spinal cord, and 1 lesion within the vertebral body.

RGCC $\leq$ II was the only independent prognostic factor related to better LC on multivariate analyses $(P$ $=0.007$; Table 2). Minimal dose $\left(\mathrm{D}_{\min }\right)$ at gross tumor volume $(\mathrm{GTV})<35$ Gy in biologically effective dose with $\alpha / \beta$ of $10\left(\mathrm{~Gy}_{10}\right)$ was associated with worse LC with marginal significance in univariate analysis $(P=0.053)$. However, there was significant correlation between RGCC and $\mathrm{D}_{\text {min }}$ at $\operatorname{GTV}(P=0.014)$, thereby, this variable was excluded from multivariate analysis. Four of 7 progressed lesions were re-irradiated with 30-50 Gy in 10 fractions. Salvage RT was done with intensity-modulation technique to minimize the spinal cord dose. Three of them achieved ultimate LC during the follow-up. The 1-year ultimate LC rates including the outcome of salvage re-irradiation were $87.2 \%$ (Figure 1B).

The 1-year OS rate was $38.9 \%$ (Figure 1C). The median survival was 7 months $(95 \%$ confidence interval, 5.03-8.97 months). On multivariate analysis, only CLIP score was identified as an independent prognostic factor related to OS $(P=0.029$; Table 2). Child-Pugh classification, which was associated with OS on univariate analysis $(P=0.011$; Table 2$)$, was excluded from the multivariate analysis because of significant correlation with CLIP score $(P=0.001)$.

\section{Post-SABR VCF and other toxicities}

Forty-two segments were evaluable for post-SABR $\mathrm{VCF}$. We observed 6 de novo VCFs and 6 progressions of pre-existing VCFs during the follow-up. Baseline spinal instability neoplastic score (SINS) components and final classification of each treated segment according to post-SABR VCF status are summarized in Table 3. The 1-year and 2-year VCF-free rates were $71.6 \%$ and $51.2 \%$, respectively (Figure 1D). The 1-year and 2-year de novo VCF-free rates were $85.4 \%$ and $61 \%$, respectively. On multivariate analysis, only pre-existing VCF was an independent prognostic factor related to VCF after SABR $(P=0.03$; Table 4). However, we observed no post-SABR VCF in patients with mixed type of lesion: therefore, lesion type, which was associated with post-VCF on univariate analysis, was excluded in the multivariate analysis. Among the segments showing post-SABR VCF, vertebroplasty was applied to 3 lesions, and fixation with screw was used for 1 lesion. 
Table 1: Patients and tumor characteristics $($ no. of patients $=29$, no. of lesions $=33$ )

Characteristics

Sex

Male

Female

Child Pugh class

A

B

CLIP score

0

1

2

3

4

5

ECOG performance scale*

0-1

2-4

Solitary bone metasiasis

Yes

No

Other metastasis

Extraspinal bone metastasis

Visceral metastasis

Tumor location

C spine

$\mathrm{C}$ and $\mathrm{T}$ spine

T spine

$\mathrm{T}$ and $\mathrm{L}$ spine

L spine

L spine and sacrum

Previous treatment to lesion of interest

Radiotherapy

Fixation

Radiation dose

$16 \mathrm{~Gy} / 1 \mathrm{fx}$

$18 \mathrm{~Gy} / 1 \mathrm{fx}$

20 Gy/1fx

$18 \mathrm{~Gy} / 3 \mathrm{fx}$
Values (\%)

28 patients $(96.5)$

1 patient (3.5)

25 patients $(86.2)$

4 patients (13.8)

7 patients $(24.1)$

5 patients (17.2)

10 patients (34.5)

5 patients (17.2)

1 patient (3.5)

1 patient (3.5)

22 patients (88.0)

3 patients (12.0)

14 patients (48.3)

15 patients $(51.7)$

11 patients (37.9)

10 patients (34.5)

5 lesions (15.2)

0 lesion (0.0)

13 lesions (39.4)

4 lesions (12.1)

10 lesions (30.3)

1 lesion (3.0)

2 lesions (6.1)

1 lesion (3.0)

3 lesions (9.1)

18 lesions (54.6)

9 lesions (27.3)

1 lesion (3.0)

(Continued) 


\begin{tabular}{lc}
\hline Characteristics & Values (\%) \\
\hline $36 \mathrm{~Gy} / 3 \mathrm{fx}$ & 1 lesion (3.0) \\
$45 \mathrm{~Gy} / 3 \mathrm{fx}$ & 1 lesion (3.0) \\
Radiographic grade of cord compression & \\
0 Spine bone involved only & 7 lesions (21.2) \\
I Thecal sac impinged & 7 lesions (21.2) \\
II Thecal sac compressed & 4 lesions (12.1) \\
III Spincal cord impinged & 10 lesions (30.3) \\
IV Cord displaced, CSF visible between cord and tumor & 5 lesions (15.2) \\
Neurological grade of cord compression ${ }^{\dagger}$ & \\
a No neurological abnormality & 18 lesions (64.3) \\
b Focal minor symptom (e.g., radiculopathy, sensory change) & 7 lesions (25.0) \\
c Functional paresis ( $\geq 4 / 5$ muscle power) & 3 lesions (10.7) \\
\hline
\end{tabular}

CLIP, Cancer of the Liver Italian Program; ECOG, Eastern Cooperative Oncology Group (ECOG); C, Cervical; T, Thoracic; L Lumbar; fx, Fraction; CSF, Cerebrospinal Fluid.

*Information for ECOG was available only for 25 patients.

tInformation for neurological grade of cord compression was available only for 28 lesions.

No patient represented radiation-induced neuropathy, even for patients who received re-irradiation during the follow-up. There was no neuropathy associated with post-SABR VCF, either. One patient, who received SABR of 20 Gy in single fraction to cervical spines, experienced headache of grade 2. One patient who received SABR of $18 \mathrm{~Gy}$ in a single fraction to thoracic spine experienced dysphagia of grade 1 .

\section{Pain and neurologic symptom responses}

The pre-SABR pain statuses were evaluable only in 23 of 33 lesions. Twenty-one of 23 lesions led to pain with numeric rating system (NRS) from 2 to 10, and 2 lesions showed no pain. Only Fifteen (45.5\%) of 33 lesions were available in analysis of pain response. The median NRS score decreased from 6 (range, 0-10) to 2 (range, 0-7) after SABR. The difference of median NRS before and after SABR was significant $(P=0.001)$. The median oral morphine equivalent dose (OMED) also decreased from $23 \mathrm{mg}$ (range, 0-155) to $15 \mathrm{mg}$ (range, 0-150) after SABR, however, the difference was not statistically significant $(P=0.564)$. Crude pain response rate according to the International Bone Metastases Consensus Group (IBMCG) was $73.3 \%$. Complete response rate and partial response rate were $33.3 \%$ and $40 \%$, respectively. Pain palliation occurred at median of 1 week (range, 1-5 weeks) after SABR. Median pain control duration of pain responders was 7 months (range, 2-8 months). One lesion showed stable pain response. This lesion presented no pain before
SABR. Therefore, no analgesic was administrated during the follow-up. Pain progression after SABR occurred in 1 lesion which accompanied progression of pre-existing VCF. Other 2 lesions showed indeterminate responses with increased OMED and decreased NRS score.

The neurologic symptom statuses before and after SABR were evaluable in 28 of 33 lesions. Among 28 lesions, 18 lesions represented neurological grade a before SABR. After SABR for these 18 lesions, the neurological grades were all stable. Among the 10 lesions representing neurologic symptoms before SABR, 7 lesions showed the neurological grade $\mathrm{b}$, and 3 lesions did the grade $\mathrm{c}$ before SABR. Among those 7 lesions with neurological grade $\mathrm{b}$, neurologic symptoms disappeared in 6 lesions $(85.7 \%)$ after SABR. However, 1 lesion represented persistent symptom of neurological grade $b$ after SABR. The median time interval between the onset of neurologic symptom and SABR of those lesions with grade $b$ was 30 days (range, 6-90 days). The time interval between the onset of neurologic symptom and SABR of the lesion with persistent neurological symptom after SABR was 21 days. Among those 3 lesions with neurological grade c, neurologic symptoms disappeared in 2 lesions $(66.7 \%)$. The median time interval between onset of symptom and SABR of those 3 lesions was 11 days (range, 10-13 days). The motor weakness was persistent in 1 lesion of which the time interval between onset of symptom and SABR was 11 days. There is no significant correlation between time interval from onset of neurologic symptom to SABR and the neurologic response. 


\section{DISCUSSION}

In the present study, we evaluated the oncologic outcome and the risk of post-SABR VCF for spinal metastasis from $\mathrm{HCC}$ which requires a high-dose irradiation, possibly by SABR, and at the same time, is vulnerable to post-SABR VCF.

In the present study, 1-year LC rate of all lesions was $68.3 \%$. For lesions with RGCC $\leq$ II, 1 -year LC rate was $92.9 \%$ which is comparable to previous results of other literatures reporting LC as $80-90 \%$ [10]. The median $\mathrm{D}_{\min }$ at GTV for lesions with RGCC $\leq$ II was $37.1 \mathrm{~Gy}_{10}$ (range, 15.6-96.0 $\mathrm{Gy}_{10}$ ). For lesions with $\mathrm{RGCC} \geq \mathrm{III}$, however, 1-year LC rate was $0 \%$, and the median $\mathrm{D}_{\min }$ at GTV in this group was only $24.8 \mathrm{~Gy}_{10}$ (range, 9.1-41.9 $\left.\mathrm{Gy}_{10}\right)$. This difference in $\mathrm{D}_{\min }$ at GTV between the 2 groups (RGCC $\leq$ II vs. $\geq$ III) resulted from the inevitable dose reduction at the area of tumor contacting with the neural structure for spinal cord sparing in the group of RGCC $\geq$ III. Nevertheless, for this group, the 1-year ultimate LC rate including the outcome of salvage re-irradiation was $67.5 \%$ which is superior to the result from conventional RT of which median LC duration was reported as 2 months $[8,9]$. Various reports have shown that the proximity of tumor to spinal cord is related to local failure in the epidural space after SABR $[11,12]$. Therefore, only tumors farther than 3-5 $\mathrm{mm}$ from the spinal cord were considered eligible for spinal SABR [13, 14]. However, under the consideration of salvage re-irradiation, SABR for spinal metastasis from HCC with RGCC $\geq$ III might be valid modality.

Although worse LC resulted from lower $\mathrm{D}_{\text {min }}$ at GTV in the present study implies the dose-response relationship of spinal metastasis from HCC, no optimal RT dose scheme for spinal metastasis from HCC has been determined. A few studies reported that SABR provided better LC comparing with conventional RT or even highdose RT of 50 Gy in 10 fractions $[8,9]$. However, largescale prospective studies are necessary to confirm the optimal dose scheme.

Among the 6 lesions showing progression in the spinal epidural space, 3 lesions initially infiltrating along the posterior longitudinal ligament presented progression in both cranial and caudal directions without involvement of adjacent vertebral bodies (Figure 2). Various studies reported no tumor progression at the immediate adjacent vertebral level near the involved vertebra $[11,15,16]$, and thereby did not recommend to include the adjacent vertebral bodies as clinical target volume (CTV) [17]. In those 3 cases, however, it is considerable to extend CTV

\section{A}

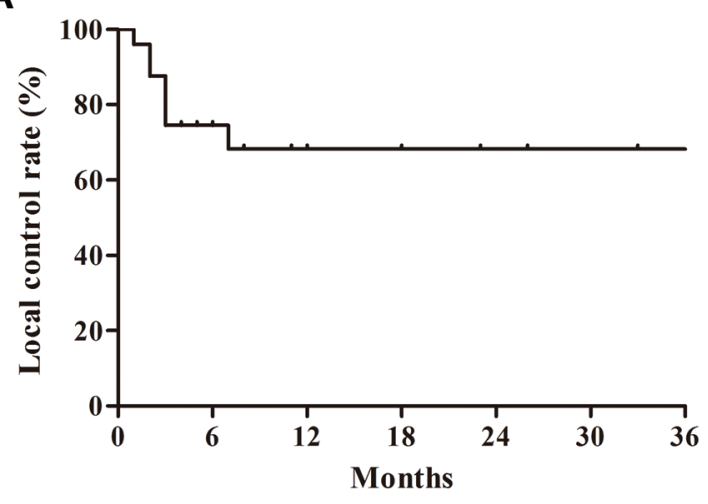

C

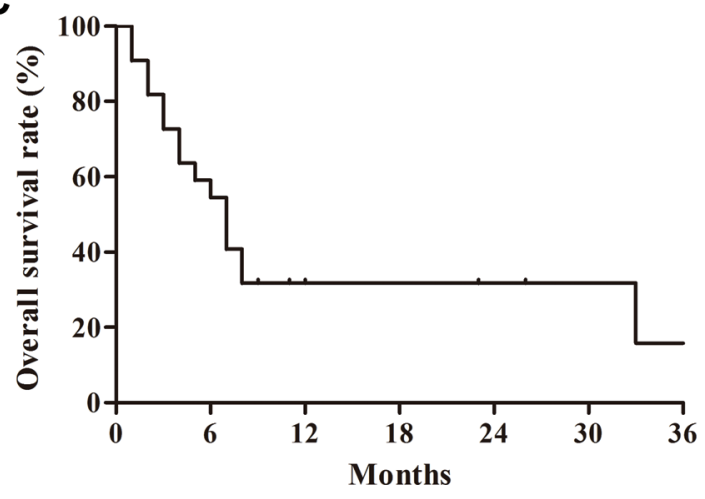

B

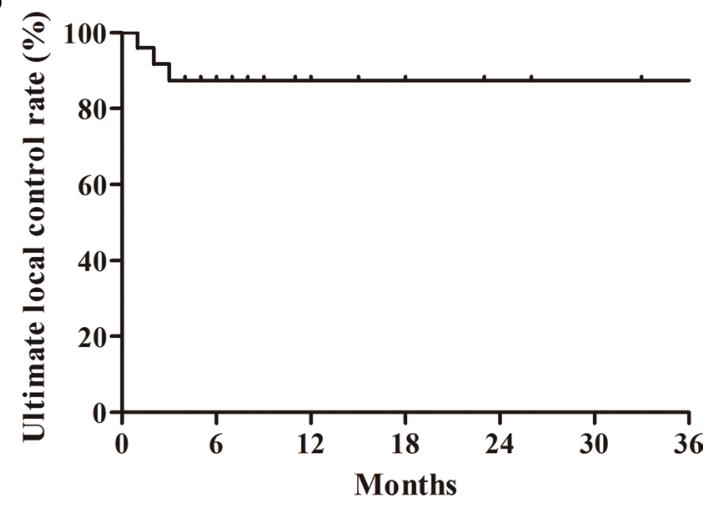

D

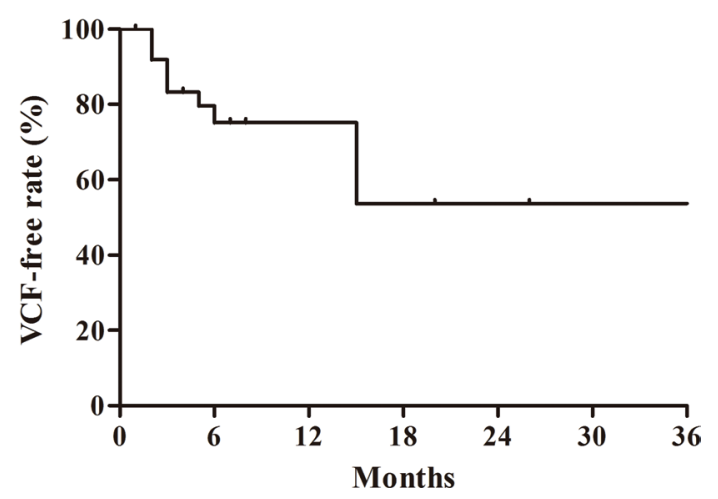

Figure 1: (A) Actuarial local control rate, (B) actuarial ultimate local control rate, (C) actuarial overall survival rate, and (D) actuarial vertebral compression fracture (VCF) free rate after stereotactic ablative body radiotherapy. 
Table 2: Univariate and multivariate analyses for local control and overall survival

\begin{tabular}{|c|c|c|c|c|c|c|}
\hline \multirow{3}{*}{ Factors } & \multicolumn{4}{|c|}{ Univariate analysis } & \multicolumn{2}{|c|}{ Multivariate analysis } \\
\hline & \multicolumn{2}{|l|}{ LC } & \multicolumn{2}{|l|}{ OS } & \multirow{2}{*}{$\frac{\text { LC }}{P \text {-value }}$} & \multirow{2}{*}{$\frac{\text { OS }}{P \text {-value }}$} \\
\hline & At 6 months $(\%)$ & $P$-value & At 6 months (\%) & $P$-value & & \\
\hline Child Pugh classification & & 0.387 & & 0.011 & NA & NA \\
\hline A & 72.1 & & 64.3 & & & \\
\hline B & 100.0 & & 25.0 & & & \\
\hline CLIP score & & 0.717 & & 0.001 & 0.260 & 0.029 \\
\hline $0-2$ & 76.2 & & 73.7 & & & \\
\hline $2-5$ & 50.0 & & 14.3 & & & \\
\hline ECOG performance scale & & 0.372 & & 0.008 & NA & 0.136 \\
\hline $0-1$ & 72.0 & & 64.2 & & & \\
\hline $2-4$ & 100.0 & & 25.0 & & & \\
\hline Solitary bone metastasis* & & 0.973 & & 0.145 & NA & NA \\
\hline No & 65.6 & & 46.7 & & & \\
\hline Yes & 64.8 & & 70.1 & & & \\
\hline Visceral metastasis & & 0.302 & & 0.052 & 0.225 & 0.083 \\
\hline No & 69.7 & & 67.1 & & & \\
\hline Yes & 85.7 & & 38.6 & & & \\
\hline BM outside spine & & 0.387 & & 0.020 & 0.617 & 0.321 \\
\hline No & 69.3 & & 71.1 & & & \\
\hline Yes & 85.7 & & 34.1 & & & \\
\hline RGCC & & 0.001 & & 0.395 & 0.007 & 0.885 \\
\hline $0-\mathrm{II}$ & 92.9 & & 68.8 & & & \\
\hline III-V & 45.0 & & 40.9 & & & \\
\hline Prescribed dose & & 0.981 & & 0.195 & 0.257 & 0.812 \\
\hline$<60 \mathrm{~Gy}_{10}$ & 71.8 & & 47.7 & & & \\
\hline$\geq 60 \mathrm{~Gy}_{10}$ & 78.8 & & 72.7 & & & \\
\hline $\mathrm{D}_{\min }$ at GTV & & 0.053 & & 0.360 & NA & NA \\
\hline$<35 \mathrm{~Gy}_{10}$ & 61.9 & & 44.8 & & & \\
\hline$\geq 35 \mathrm{~Gy}_{10}$ & 90.0 & & 80.0 & & & \\
\hline
\end{tabular}

LC, Local Control; OS, Overall Survival; NA, Not Analyzed; CLIP, Cancer of the Liver Italian Program; ECOG, Eastern Cooperative Oncology Group; BM, Bone Metastasis; RGCC, Radiographic Grade of Cord Compression; $\mathrm{Gy}_{10}$, Gy of Biologically Effective Dose with $\alpha / \beta=10 ; D_{\text {min }}$, Minimal Dose; GTV, Gross Tumor Volume.

*Solitary bone metastasis was excluded in multivariate analysis because of its significant correlations with visceral metastasis $(P<0.001)$ and BM outside spine $(P<0.001)$.

in both cranial and caudal directions for encompassing the adjacent area along the posterior longitudinal ligament to reduce the risk of local failure.

In the present study, the 1-year VCF-free rate was $71.6 \%$. In other studies, the VCF-free rate after SABR was reported to be $61-81 \%[7,18,19]$. Few studies, however, have evaluated the risk of VCF after SABR for spinal metastasis from HCC. And also those studies have very small sample size, or reported VCF rate containing the outcome of various RT regimens other than $\operatorname{SABR}[7,8]$. 
Table 3: Scores according to SINS component and final classification of each treated vertebral segment

\begin{tabular}{|c|c|c|c|}
\hline Factor & $\begin{array}{c}\text { Post-SABR VCF } \\
(\mathrm{N}=12 \text { segments })\end{array}$ & $\begin{array}{l}\text { No Post-SABR VCF } \\
(\mathbf{N}=\mathbf{3 0} \text { segments })\end{array}$ & $\%$ of $\mathrm{VCF}$ \\
\hline \multicolumn{4}{|l|}{ Location } \\
\hline Junctional & 5 & 15 & 25.0 \\
\hline Mobile & 4 & 8 & 33.3 \\
\hline Semi-rigid & 3 & 7 & 30.0 \\
\hline Rigid & 0 & 0 & - \\
\hline \multicolumn{4}{|l|}{ Pain } \\
\hline Mechanical & 8 & 20 & 28.6 \\
\hline $\begin{array}{l}\text { Occasional and non- } \\
\text { mechanical }\end{array}$ & 1 & 5 & 16.7 \\
\hline None & 3 & 5 & 37.5 \\
\hline \multicolumn{4}{|l|}{ Bone lesion type } \\
\hline Only-lytic & 12 & 20 & 37.5 \\
\hline Mixed (lytic and blastic) & 0 & 10 & 0.0 \\
\hline \multicolumn{4}{|l|}{ Alignment } \\
\hline Subluxation/translation & 1 & 0 & 100.0 \\
\hline Kyphosis/scoliosis & 0 & 0 & - \\
\hline Normal & 11 & 30 & 36.7 \\
\hline \multicolumn{4}{|l|}{ Vertebral body collapse } \\
\hline$\geq 50 \%$ & 2 & 0 & 100.0 \\
\hline$<50 \%$ & 4 & 4 & 100.0 \\
\hline $\begin{array}{l}\text { No collapse by }>50 \% \text { of the } \\
\text { bodies affected by tumor }\end{array}$ & 1 & 2 & 33.3 \\
\hline None of the above & 5 & 24 & 17.2 \\
\hline \multicolumn{4}{|l|}{ Posterior element involvement } \\
\hline Bilateral & 1 & 1 & 50.0 \\
\hline Unilateral & 0 & 5 & 0.0 \\
\hline Not involved & 11 & 24 & 31.4 \\
\hline \multicolumn{4}{|l|}{ SINS } \\
\hline Stable $(0-6)$ & 3 & 16 & 15.8 \\
\hline $\begin{array}{l}\text { Indeterminate instability (7- } \\
\text { 12) }\end{array}$ & 8 & 14 & 36.4 \\
\hline Unstable (13-) & 1 & 0 & 100.0 \\
\hline
\end{tabular}

SABR, Stereotactic Ablative Body Radiotherapy; VCF, Vertebral Compression Fracture; SINS, Spinal Instability Neoplastic Score.

To our knowledge, the present study is the first to report the risk of VCF after SABR for spinal metastases from HCC specifically. The results showed the accordance with other studies that identified pre-existing VCF and osteolytic change as significant factors of post-SABR VCF $[7,18,19]$. Considerable post-VCF rates in our study can be explained by soft-tissue formation and the osteolytic nature of spinal metastasis from $\mathrm{HCC}[4,5,20]$. Osteolytic 
Table 4: Univariate and multivariate analyses for vertebral compression fracture-free survival

\begin{tabular}{|c|c|c|c|c|}
\hline \multirow{2}{*}{ Variable } & \multicolumn{2}{|c|}{ Univariate analysis } & \multicolumn{2}{|c|}{ Multivariate analysis } \\
\hline & 1yr-VCFFR (\%) & $P$-value & HR (95\% CI) & $P$-value \\
\hline \multicolumn{5}{|l|}{ Baseline SINS } \\
\hline$\leq 6$ & 87.4 & 0.037 & $0.453(0.074-2.765)$ & 0.391 \\
\hline$>6$ & 59.8 & & & \\
\hline \multicolumn{5}{|l|}{ Lesion type } \\
\hline Mixed type & 100.0 & 0.017 & NA & NA \\
\hline Lytic only & 61.8 & & & \\
\hline \multicolumn{5}{|l|}{ Previous VCF before SABR } \\
\hline No & 85.4 & $<0.001$ & $0.199(0.046-0.859)$ & 0.030 \\
\hline Yes & 22.5 & & & \\
\hline \multicolumn{5}{|l|}{ Maximum dose on segment } \\
\hline$<20$ Gy & 74.5 & 0.689 & $0.814(0.236-2.803)$ & 0.744 \\
\hline$\geq 20 \mathrm{~Gy}$ & 70.4 & & & \\
\hline \multicolumn{5}{|l|}{ Pain } \\
\hline Mechanical & 72.0 & 0.872 & $1.171(0.319-4.301)$ & 0.813 \\
\hline Non-mechanical or none & 68.1 & & & \\
\hline
\end{tabular}

VCFFR, Vertebral Compression Fracture-Free Rate; HR, Hazard Ratio; 95\% CI, 95\% Confidence Interval; SINS, Spinal Instability Neoplastic Score; NA, Not Analyzed; VCF, Vertebral Compression Fracture; SABR, Stereotactic Ablative Body Radiotherapy.

change makes vertebra weaker to compressive stress. And the shrinkage of metastatic soft-tissue after SABR could lead to vertebral collapse, because the soft-tissue mass itself provides resistance to the compressive stress in the involved vertebra. In the present study, $33.3 \%$ of segments with post-SABR VCF required an intervention. Therefore, early preventive vertebroplasty is considerable, especially for segments with pre-existing VCF or only-lytic changes.

In the present study, there is no observed radiationinduced neurotoxicity even in the patients who received salvage re-irradiation. This may be due to the RT planning protocol which gives priority to spinal cord dose constraint rather than GTV dose for spinal cord saving. However, whether 7 months of follow-up duration is sufficient to discuss the radiation-induced neuropathy is questionable. The latency periods of neuropathy after RT have been reported in wide range, mainly from one to several years [21]. For SABR, on the other hand, shorter latency period ( 0.6 year) of radiation-induced neuropathy was reported although the data is very scares [22]. Therefore, the follow-up period might be sufficient to discuss the radiation-induced neuropathy, although longer followup duration may be necessary to confirm the risk for radiation-induced neuropathy of SABR.
The pain response rates of spine SABR have been reported as $67-100 \%$ with various doses [14]. However, those rates were not under consideration of the confounding effect of analgesics. After adjustment of analgesic effect, pain response rates after RT with various doses were reported as only $37-50 \%$ [23]. In the present study, the pain response rate according to criteria of IBMCG was $73.3 \%$ with median pain control duration of 7 months after SABR. The median NRS also decreased after SABR significantly. Although prospective result or randomized trial is necessary, SABR might be more efficacious in pain relief and durability of pain control than RT of high-dose in various fractionation, or even 8 Gy in single fraction which showed median duration of pain control as 3 months [5, 24, 25], especially for the patients to whom higher-dose RT is recommended.

Considering that the aim of spinal SABR is longterm LC and pain-control without post-SABR VCF, it is important to select the appropriate patients expected to be long-term survivors without any morbidities. The prognosis of HCC depends on both tumor controllability and residual liver function, which are covered in the CLIP scoring system [26]. Our study identified CLIP score as an independent predictor of OS. Therefore, optimal outcome 
is expected to be achieved for patients with CLIP $\leq 2$, the spine metastasis of RGCC $\leq \mathrm{II}$, and neither pre-existing VCF nor only-osteolytic lesion.

There are some limitations in the present study. There is an inherent bias, because the present study was a retrospective one from a single institute with a small sample size. Especially, the small number of medical records available for the analysis of pain response was a significant limitation. Another limitation is related to the systemic modality, especially sorafenib. Although our study showed no significant association between use of sorafenib and oncologic outcome, confounding effects could not be excluded.

In conclusion, SABR for spinal metastases from $\mathrm{HCC}$ is effective for pain relief and durable pain control. It is also efficacious in LC, especially for spinal metastases with RGCC $\leq$ II. Although SABR for spinal metastases with $\mathrm{RGCC} \geq \mathrm{III}$ showed poor LC, salvage re-irradiations are feasible. Because patients with normal liver function and controllable hepatic tumor status are expected to be long-term survivors, selection of appropriate patients can lead to achieve long-term survival without disease progression. As post-SABR VCF occurred frequently for vertebral segments with pre-existing VCF and only-lytic change, early intervention is considerable for those high-risk segments. SABR is a potentially valid modality for spinal metastases from HCC.

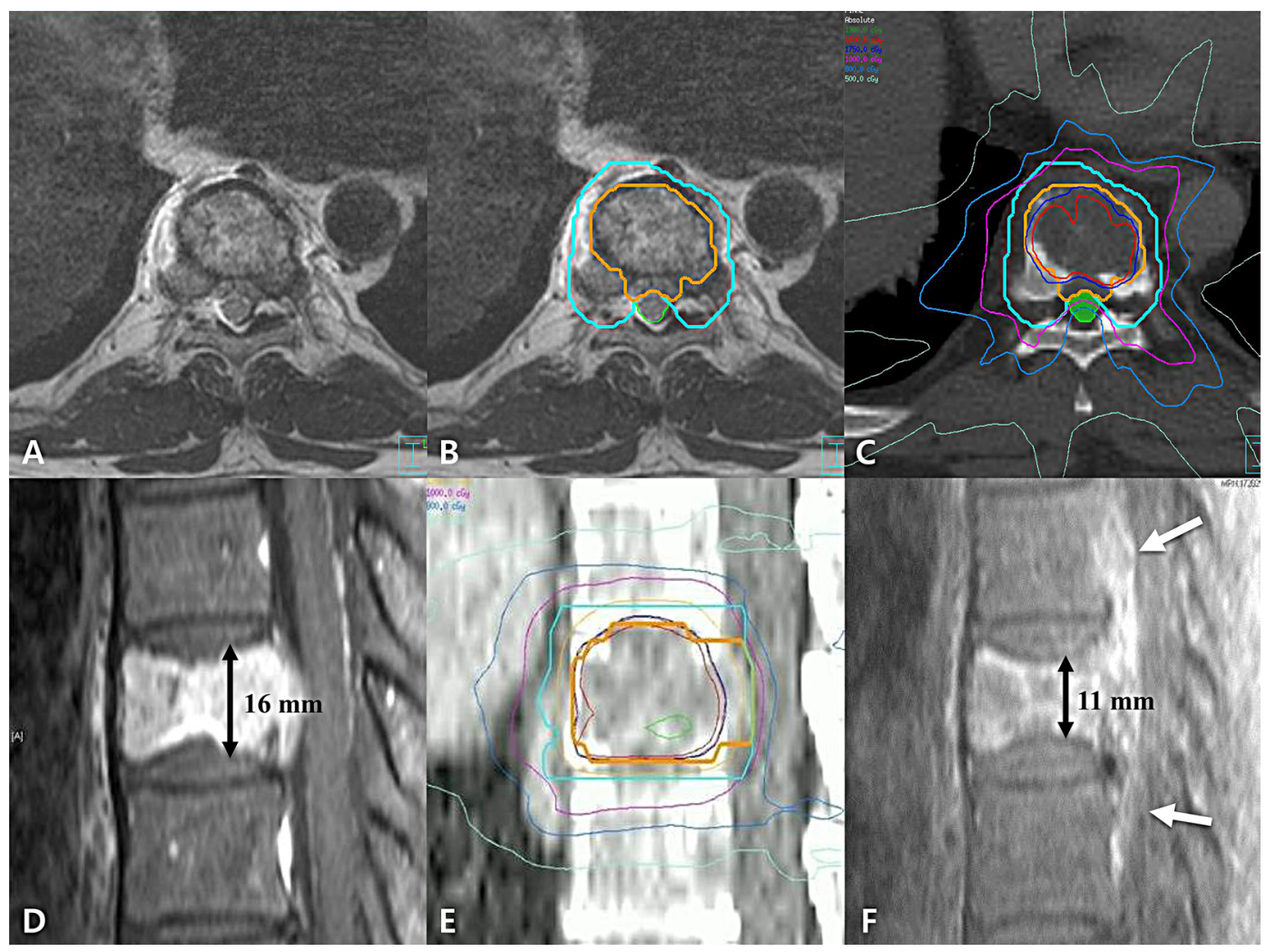

Figure 2: (A) Axial view of planning magnetic resonance image (MRI), (B) example of target delineation in axial view, (C) example of dose distribution in axial view, (D) sagittal view of planning MRI, (E) example of target delineation and dose distribution in sagittal view, (F) sagittal view of follow-up MRI at 3 months after stereotactic ablative body radiotherapy (SABR). The patient, who was 60 -year-old male with spinal metastasis with radiographic grade of cord compression III, received SABR of 18 Gy in 1 fraction. Gross tumor volume (GTV), clinical target volume, and spinal cord was delineated in orange, sky blue, and green color, respectively. Because the GTV contacts with spinal cord, we concerned the spinal cord delineation more than GTV delineation. The GTV and spinal cord volume are exclusive to each other (B). The spinal cord dose constraint was also more concerned rather than GTV dose for spinal cord saving (C). Before SABR, we observed the tumor infiltration along the posterior longitudinal ligament (PLL) (D). The tumor progression was shown in both cranial and caudal directions along the PLL at 3 months after SABR (white arrow; F). The patient had pre-existing vertebral compression fracture (VCF) with Spinal Instability Neoplatic Score of 8 (D). There is decrease in height of vertebra body from $16 \mathrm{~mm}$ to $11 \mathrm{~mm}$ at $3 \mathrm{months}$ after SABR which means the progression of pre-existing VCF $(\mathrm{F})$. 


\section{MATERIALS AND METHODS}

\section{Patients}

Thirty-three lesions in 42 spinal segments from 29 patients were reviewed retrospectively. Patients of the study were diagnosed with spinal metastasis from HCC and received SABR for spinal metastasis between September 2009 and January 2015. Patients were diagnosed by computed tomography (CT), magnetic resonance imaging (MRI), positron emission tomography (PET)/CT, or biopsy. Initial severity of spinal cord compression was evaluated by the RGCC system proposed by S. Ryu [27, 28]. Baseline instabilities of spinal segments were evaluated according to the SINS developed by the Spine Oncology Study Group [29]. Initial pain status was assessed with NRS score. Neurological symptoms were graded by neurological grade system suggested by Ryu S et al [27, 28]. Inclusion criteria for the study group were (1) spinal metastasis with involvement of 1-3 spinal segments, (2) ambulant status, (3) ChildPugh classification A or B, and (4) no requirement for immediate stabilization of the spine.

\section{Treatments}

All patients underwent simulation with both $\mathrm{CT}$ and MRI scans sequentially with immobilization system. Slice thickness of CT scan was $2.5 \mathrm{~mm}$. T1- and T2-weighted images were obtained during simulation MRI scanning, and fused with the simulation CT images.

GTV and CTV were delineated according to the Radiation Therapy Oncology Group (RTOG) 0631 protocol [13]. There was no margin expansion between CTV and planning target volume. Normal organs near the target were delineated for evaluation of dose of organs-atrisk (OARs). If the tumor contacts with spinal cord, we concerned the spinal cord delineation more than tumor volume delineation. The GTV and OAR volume are exclusive to each other.

The prescription doses were 16-20 Gy in 1-fraction, or 18-45 Gy in 3-fractions. Dose-constraints of OARs also followed the RTOG 0631 protocol. We intended to deliver a prescribed dose covering at least $90 \%$ of the GTV; if this was not possible due to spinal cord dose, we gave priority to spinal cord dose constraint rather than GTV dose. We used intensity-modulation with an inverse planning method. Every SABR was performed by Novalis $\mathrm{Tx}^{\mathrm{TM}}$ (Branilab AG, Heimstentten, Germany). For verification of setup, cone-beam CT and Exactrac were used before each treatment.

\section{Evaluation of treatment response and toxicity}

Patients were followed with CT, MRI, or PET/CT every 1-3 months after SABR. We defined LC as neither progressive disease nor metabolic progressive disease status according to revised Response Evaluation Criteria in Solid Tumors criteria [30], or PET Response Criteria in Solid Tumors criteria [31]. Local failure was defined as progression within the $75 \%$ isodose line. LC duration was the time interval from the SABR to time of disease progression. We censored the patients who did not show local failure at the date of last visit. OS duration was defined as the interval between the SABR and time of death, or last visit if the patient was alive.

We assessed the pain response by change in NRS score at 1 week after SABR and every 1-3 months. To adjust the confounding effects of analgesics, we evaluated the pain response according to categories of the IBMCG [32]. To apply these categories, we calculated the OMED of all analgesics administrated to patients before and after the SABR.

We defined post-SABR VCF as new development of de novo $\mathrm{VCF}$ or progression of pre-existing $\mathrm{VCF}$ shown as shortening of vertebral body height after SABR. VCF-free duration is the time to VCF from SABR or last follow-up date if no VCF was shown. We evaluated other complications according to the Common Terminology Criteria for Adverse Events, version 4.0.1.

\section{Statistical analysis}

Descriptive statistics were used to assess patient and tumor characteristics. LC rate, OS rate, and VCF-free rate were estimated by the Kaplan-Meier method. Univariate analysis was performed by log-rank test for potential predictors. A multivariate Cox regression model was applied to determine the independent prognostic factors associated with LC, OS, and VCF-free rate. For any 2 variables significantly correlated with each other, one of the variables was excluded from multivariate analysis. Any variables showing no event were also excluded from multivariate analysis. Mann-Whitney test was applied to evaluate the significance of the change in NRS and OMED before and after SABR. All $P$-values in this report were two-sided. Results were considered significant if $P<0.05$. We used SPSS version 22 (IBM, Armonk, NY, USA) for all statistical analyses.

\section{Abbreviations}

HCC, Hepatocellular Carcinoma; OS, Overall Survival; RT, Radiotherapy; SABR, Stereotactic Ablative Body Radiotherapy; VCF, Vertebral Compression Fracture; LC, Local Control; CLIP, Cancer of the Liver Italian Program; RGCC, Radiographic Grade of Cord Compression; $\mathrm{D}_{\min }$, Minimal Dose; GTV, Gross Tumor Volume; $\mathrm{Gy}_{10}$, Biologically Effective Dose with $\alpha / \beta$ of 10 ; SINS, Spinal Instability Neoplastic Score; NRS, Numeric Rating System; OMED, Oral Morphine Equivalent Dose; IBMCG, International Bone Metastases Consensus Group; 
CTV, Clinical Target Volume; CT, Computed Tomography; MRI, Magnetic Resonance Imaging; PET, Positron Emission Tomography; RTOG, Radiation Therapy Oncology Group; OAR, Organ at Risk; ECOG, Eastern Cooperative Oncology Group; BM, Bone Metastasis; VCFFR, Vertebral Compression Fracture-Free Rate; HR, Hazard Ratio; CI, 95\% Confidence Interval; NA, Not Analyzed.

\section{Author contributions}

GS Yoo performed the analysis of the data and wrote this manuscipt. HC Park designed this research and revised this article. JI Yu and SJ Park revised and finally approved the article to be published. Other authors revised this article.

\section{ACKNOWLEDGMENTS}

We specially thanks to Su Yeon Park's devotion to RT dosimetry and restoration of RT planning.

\section{CONFLICTS OF INTEREST}

The authors declare no conflicts of interest.

\section{FUNDING}

This research was supported by a grant from Samsung Medical Center (SMX1170051), and a grant from the Marine Biotechnology Program (20150220) funded by the Ministry of Oceans and Fisheries, Korea.

\section{REFERENCES}

1. Lafaro KJ, Demirjian AN, Pawlik TM. Epidemiology of hepatocellular carcinoma. Surg Oncol Clin N Am. 2015; 24:1-17.

2. Chang SS, Luo JC, Chao Y, Chao JY, Chi KH, Wang SS, Chang FY, Lee SD, Yen SH. The clinical features and prognostic factors of hepatocellular carcinoma patients with spinal metastasis. Eur J Gastroenterol Hepatol. 2001; 13:1341-1345.

3. Cho HS, Oh JH, Han I, Kin HS. Survival of patients with skeletal metastases from hepatocellular carcinoma after surgical management. J Bone Joint Surg Br. 2009; 91:1505-12.

4. He J, Zeng ZC, Tang ZY, Fan J, Zhou J, Zeng MS, Wang JH, Sun J, Chen B, Yang P, Pan BS. Clinical features and prognostic factors in patients with bone metastases from hepatocellular carcinoma receiving external beam radiotherapy. Cancer. 2009; 115:2710-2720.

5. Seong J, Koom WS, Park HC. Radiotherapy for painful bone metastases from hepatocellular carcinoma. Liver Int. $2005 ; 25: 261-265$.
6. Nakamura N, Igaki H, Yamashita H, Shiraishi K, Tago M, Sasano N, Shiina S, Omata M, Makuuchi M, Ohtomo K, Nakagawa K. A retrospective study of radiotherapy for spinal bone metastases from hepatocellular carcinoma (HCC). Jpn J Clin Oncol. 2007; 37:38-43.

7. Cunha MV, Al-Omair A, Atenafu EG, Masucci GL, Letourneau D, Korol R, Yu E, Howard P, Lochray F, da Costa LB, Fehlings MG, Sahgal A. Vertebral compression fracture (VCF) after spine stereotactic body radiation therapy (SBRT): analysis of predictive factors. Int J Radiat Oncol Biol Phys. 2012; 84:e343-e349.

8. Lee E, Kim TG, Park HC, Yu JI, Lim do H, Nam H, Lee H, Lee JH. Clinical outcomes of stereotactic body radiotherapy for spinal metastases from hepatocellular carcinoma. Radiat Oncol J. 2015; 33:217-225.

9. Chang UK, Kim MS, Han CJ, Lee DH. Clinical result of stereotactic radiosurgery for spinal metastasis from hepatocellular carcinoma: comparison with conventional radiation therapy. J Neurooncol. 2014; 119:141-148.

10. Sahgal A, Bilsky M, Chang EL, Ma L, Yamada Y, Rhines LD, Letourneau D, Foote M, Yu E, Larson DA, Fehlings MG. Stereotactic body radiotherapy for spinal metastases: current status, with a focus on its application in the postoperative patient. J Neurosurg Spine. 2011; 14:151-166.

11. Chang EL, Shiu AS, Mendel E, Mathews LA, Mahajan A, Allen PK, Weinberg JS, Brown BW, Wang XS, Woo SY, Cleeland C, Maor MH, Rhines LD. Phase I/II study of stereotactic body radiotherapy for spinal metastasis and its pattern of failure. J Neurosurg Spine. 2007; 7:151-160.

12. Bishop AJ, Tao R, Rebueno NC, Christensen EN, Allen PK, Wang XA, Amini B, Tannir NM, Tatsui CE, Rhines LD, Li J, Chang EL, Brown PD, et al. Outcomes for spine stereotactic body radiation therapy and an analysis of predictors of local recurrence. Int J Radiat Oncol Biol Phys. 2015; 92:1016-1026.

13. Ryu S, Pugh SL, Gerszten PC, Yin FF, Timmerman RD, Hitchcock YJ, Movsas B, Kanner AA, Berk LB, Followill DS, Kachnic LA. RTOG 0631 phase II/III study of imageguided stereotactic radiosurgery for localized (1-3) spine metastases: phase II results. Int J Radiat Oncol Biol Phys. 2011; 81:S131-S132.

14. Sahgal A, Larson DA, Chang EL. Stereotactic body radiosurgery for spinal metastases: a critical review. Int $\mathrm{J}$ Radiat Oncol Biol Phys. 2008; 71:652-665.

15. Gerszten PC, Burton SA, Ozhasoglu C, Welch WC. Radiosurgery for spinal metastases: clinical experience in 500 cases from a single institution. Spine (Phila Pa 1976). 2007; 32:193-199.

16. Ryu S, Rock J, Rosenblum M, Kim JH. Patterns of failure after single-dose radiosurgery for spinal metastasis. J Neurosurg. 2004; 101:402-405.

17. Cox BW, Spratt DE, Lovelock M, Bilsky MH, Lis E, Ryu S, Sheehan J, Gerszten PC, Chang E, Gibbs I, Soltys S, Sahgal A, Deasy J, et al. International Spine Radiosurgery Consortium consensus guidelines for target volume 
definition in spinal stereotactic radiosurgery. Int J Radiat Oncol Biol Phys. 2012; 83:e597-e605.

18. Sahgal A, Atenafu EG, Chao S, Al-Omair A, Boehling N, Balagamwala EH, Cunha M, Thibault I, Angelov L, Brown P, Suh J, Rhines LD, Fehlings MG, et al. Vertebral compression fracture after spine stereotactic body radiotherapy: a multi-institutional analysis with a focus on radiation dose and the spinal instability neoplastic score. J Clin Oncol. 2013; 31:3426-3431.

19. Al-Omair A, Smith R, Kiehl TR, Lao L, Yu E, Massicotte EM, Keith J, Fehlings MG, Sahgal A. Radiation-induced vertebral compression fracture following spine stereotactic radiosurgery: clinicopathological correlation. J Neurosurg Spine. 2013; 18:430-435.

20. Kim S, Chun M, Wang H, Cho S, Oh YT, Kang SH, Yang J. Bone metastasis from primary hepatocellular carcinoma: characteristics of soft tissue formation. Cancer Res Treat. 2007; 39:104-108.

21. Delanian S, Lefaix JL, Pradat PF. Radiation-induced neuropathy in cancer survivors. Radiother Oncol. 2012; 105:273-282.

22. Forquer JA, Fakiris AJ, Timmerman RD, Lo SS, Perkins SM, McGarry RC, Johnstone PA. Brachial plexopathy from stereotactic body radiotherapy in early-stage NSCLC: doselimiting toxicity in apical tumor sites. Radiother Oncol. 2009; 93:408-413.

23. Zaikova O, Fossa SD, Kongsgaard U, Kvaloy S, Giercksky KE, Skjeldal S. Pain after palliative radiotherapy for spine metastases. Clin Oncol (R Coll Radiol). 2010; 22:828-836.

24. Hayashi S, Tanaka H, Hoshi H. External beam radiotherapy for painful bone metastases from hepatocellular carcinoma: multiple fractions compared with an 8-Gy single fraction. Nagoya J Med Sci. 2014; 76:91-99.

25. Hartsell WF, Scott CB, Bruner DW, Scarantino CW, Ivker RA, Roach M 3rd, Suh JH, Demas WF, Movsas B, Petersen IA, Konski AA, Cleeland CS, Janjan NA, et al. Randomized trial of short-versus long-course radiotherapy for palliation of painful bone metastases. J Natl Cancer Inst. 2005; 97:798-804.

26. Kudo M, Chung H, Osaki Y. Prognostic staging system for hepatocellular carcinoma (CLIP score): its value and limitations, and a proposal for a new staging system, the Japan Integrated Staging Score (JIS score). J Gastroenterol. 2003; 38:207-215.

27. Ryu S, Rock J, Jain R, Lu M, Anderson J, Jin JY, Rosenblum M, Movsas B, Kim JH. Radiosurgical decompression of metastatic epidural compression. Cancer. 2010; 116:2250-2257.

28. Ryu S, Yoon H, Stessin A, Gutman F, Rosiello A, Davis R. Contemporary treatment with radiosurgery for spine metastasis and spinal cord compression in 2015. Radiat Oncol J. 2015; 33:1-11.

29. Fisher CG, DiPaola CP, Ryken TC, Bilsky MH, Shaffrey CI, Berven SH, Harrop JS, Fehlings MG, Boriani S, Chou D, Schmidt MH, Polly DW, Biagini R, et al. A novel classification system for spinal instability in neoplastic disease: an evidence-based approach and expert consensus from the Spine Oncology Study Group. Spine (Phila Pa 1976). 2010; 35:E1221-E1229.

30. Eisenhauer EA, Therasse P, Bogaerts J, Schwartz LH, Sargent D, Ford R, Dancey J, Arbuck S, Gwyther S, Mooney M, Rubinstein L, Shankar L, Dodd L, et al. New response evaluation criteria in solid tumours: revised RECIST guideline (version 1.1). Eur J Cancer. 2009; 45:228-247.

31. Wahl RL, Jacene H, Kasamon Y, Lodge MA. From RECIST to PERCIST: evolving considerations for PET response criteria in solid tumors. J Nucl Med. 2009; 50:122S-150S.

32. Chow E, Hoskin P, Mitera G, Zeng L, Lutz S, Roos D, Hahn C, van der Linden Y, Hartsell W, Kumar E; International Bone Metastases Consensus Working Party. Update of the international consensus on palliative radiotherapy endpoints for future clinical trials in bone metastases. Int $\mathrm{J}$ Radiat Oncol Biol Phys. 2012; 82:1730-1737. 